\title{
DIFFUSION OF LOW ENERGY AURORAL ELECTRONS IN THE ATMOSPHERE
}
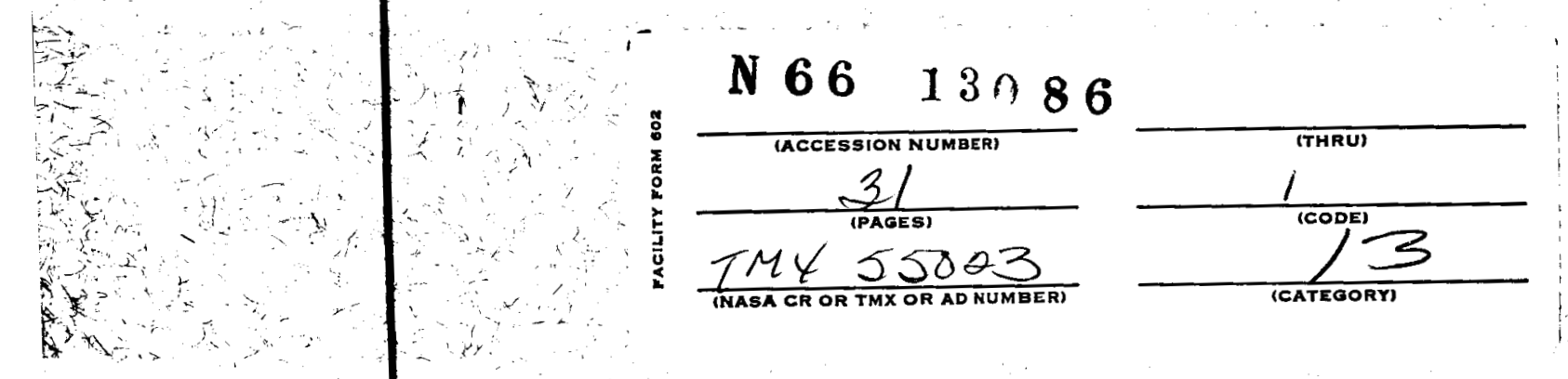

\section{IUNE 1964}

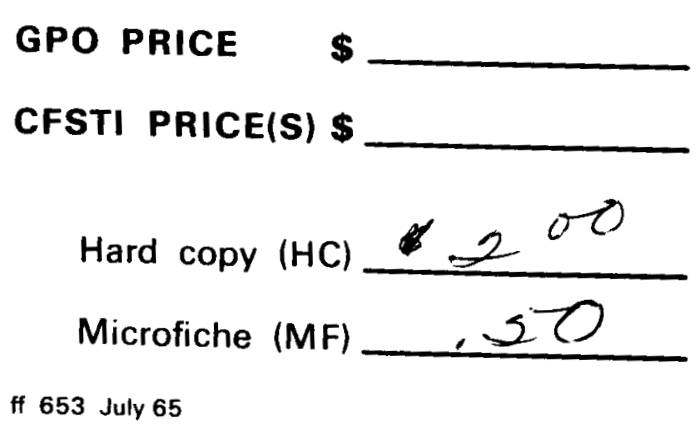




\title{
DIFFUSION OF LOW ENERGY AURORAL ELECTRONS \\ IN THE ATMOSPHERE
}

by

Kaichi Maeda

NASA-Goddard Space Flight Center

Greenbelt, Maryland

\begin{abstract}
136) 8C.

By means of the Monte Carlo method, Spencer's work on the energy dissipation of electrons in air is extended below $25 \mathrm{Kev}$, and the results are applied to the diffusion of mono-energetic electrons in the polar atmosphere. The results show significant effects of straggling, not only on the cistribution of the most penetrating flux but also on the height of maximum dissipation and on the back-scattering. The dependence of back-scattering on the angular distributions as well as the energies of incident electrons are calculated, indicating that it decreases rapidly below $10 \mathrm{Kev}$ and that a maximum of back-scattering seems to occur at energies around $20 \mathrm{Kev}$, with values of the order of $7 \%$ and $20 \%$ for vertical and isotropic incidence, respectively for intensity. Corresponding energy back-scattering coefficients (albedos) are approximately half of these values.

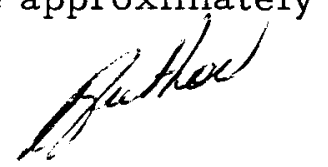




\title{
DIFFUSION OF LOW ENERGY AURORAL ELECTRONS \\ IN THE ATMOSPHERE
}

\author{
1. Introduction
}

In calculating the rates of reactions caused by auroral activities (such as the vertical distributions of luminosity and auroral absorption or the rate of dissociation of air due to bombarding auroral particles) it is necessary to have fundamental information on the behavior of primary auroral particles in the upper atmosphere. This information includes changes of intensity (particle flux) and of energy flux, and the rate of these changes with atmospheric depth.

Actually the se can be calculated easily with the required accuracy by using range-energy relations for protons but not for electrons. (Rossi, 1952; Maeda and Singer, 1961; Maeda, 1962, 1963 a,b). Because of the dominating effect of Coulomb scattering and its statistical nature in collision processes, no rigorous calculations are available for these purposes, except for an extensive computation on the energy dissipation of electrons done by V. L. Spencer (1959).

Recently, M. H. Rees (1963) made semi-empirical calculations on the luminosity distribution of aurora, which are based on the laboratory data given by A. E. Grün (1957). Since Grün's measurements are limited in the case of the vertical incidence of mono-energetic electrons with energies between 5 and $54 \mathrm{Kev}$, Rees had to make some ad hoc assumptions on angular distribution in order to extend Grü's results to other cases. 
The purpose of the present calculations is, therefore, as follows:

(1) To extend Spencer's work below $25 \mathrm{Kev}$, because most auroral electrons are in this energy range.

(2) To find not only the energy dissipation rates in air and the amount of back-scattering but also the changes of angular distributions and of energy spectra with depth due to scattering and straggling.

(3) To apply the results to the diffusion of mono-energetic auroral electrons in the atmosphere.

The importance of the straggling effect on penetrating electrons has been mentioned in several papers concerning the tail part of intensity and of the energy distribution of electrons (Spencer, 1959; Grün, 1957; Perkins, 1962; Rees, 1963). These tailing distributions are due to both scattering and fluctuation in the energy loss rate at each collision. Therefore, sometimes the word "straggling" is used for those combined effects. In this paper, however, the straggling effect means only the effect due to fluctuations in energy loss, namely, the effect due only to Landau fluctuation (1944).

It should be noted that Spencer's calculation, which is based on the so-called moments method, cannot take the straggling effect into account. At present the only way to take these effects, as well as multiple Coulomb scattering and continuous energy loss, into consideration simultaneously is by the Monte Carlo method.

Since the geomagnetic field is nearly vertical and uniform in the polar region, the unwinding of the spiraling path of impinging electrons 
is equivalent to the oblique path with the incident angle equal to the pitch angle. Therefore, the results of computations which are performed with respect to the air, assuming uniform density without a magnetic field, are applied to the electrons diffusing into the polar upper atmosphere by using the CIRA atmospheric model (Kallmann-Bijl, et al, 1961).

\section{Calculations}

The Monte Carlo calculation consists essentially of two parts, namely, the basic data which contain formulas for scattering and energy loss as a function of the energy of electrons in the medium (i.e., in air), and the random sampling for combinations of these basic data for a given number of histories.

The programs for machine calculation have been made at several places for different purposes. A review on this type of calculation is given by M. J. Berger (1963). Details of the program (Fortran for IBM 7090) used in the present calculation have been published by Berger (1963). Similar reports also have been written recently by Berger and Seltzer (1964 a, b).

The formulas used for the basic data are as follows:

(1) Energy loss. Since the energies of electrons in the present calculation are below $25 \mathrm{Kev}$, radiation losses are completely neglected. Electron trajectories are schematically divided into a number of short sections, whose lengths are chosen so that a mean energy loss in each section corresponds to a certain factor $\left(2^{-1 / 16}\right.$ in most cases) of 
reduction of the electron energy. The mean energy loss is calculated according to the Bethe theory formulated by Rohrlich and Carlson (1954).

(2) Angular deflection. For the angular distribution, the multiple scattering theory of Goudsmit and Saunderson (1940) with Mott's cross section for single scattering (Mott, 1929) is used instead of the familiar Moliere's theory for multiple Coulomb scattering (Moliere, 1948). This gives better accuracy for a large deflection.

(3) Straggling. The expression for fluctuation of the energy loss from a continuous loss rate is taken from the paper by Blunck and Leisegang (1950) instead of using the original expression by Landau (1944).

The basic quantity to be computed is

$$
\mathrm{T}\left(\mathrm{E}_{0}, \cos \theta_{0} \mathrm{E}, \cos \theta, \mathbf{x}\right) \mathrm{dE} \cdot \mathrm{d} \cos \theta .
$$

This is called the differential transmission (Berger, 1963). It represents the probability that an electron with initial energy $\mathbf{E}_{0}$ at an incident angle $\theta_{0}$ from the normal direction will appear at a depth $\mathbf{x}$ energy with $\mathrm{E}$ at an angle $\theta$ (between $\cos \theta$ and $\cos \theta+\mathrm{d} \cos \theta$ ). The depth $\mathbf{x}$ is expressed by the units of range $r_{0}$, i.e., $x=r / r_{0}$ where $r$ is the thickness of the layer in $\mathrm{g} / \mathrm{cm}^{2}$ and $\mathrm{r}_{0}$ is

$$
r_{0}=\int_{0}^{E_{0}} d E /\left(-\frac{d E}{d x}\right)
$$


The Bethe formula is used for $-\mathrm{dE} / \mathrm{dx}$. Range $\mathrm{r}_{0}$ in $\mathrm{g} \mathrm{cm}^{-2}$ and height $Z_{0}$ in CIRA (1961) model corresponding $r_{0}$ for given $E_{0}$ are shown in Table 1 .

The other quantities of interest are obtained by summing the differential transmission as follows:

(1) The relative energy spectrum at $\mathbf{x}$ is

$$
i\left(E_{0}, E, x\right)=\int_{0}^{1} T\left(E_{0}, E, \cos \theta, x\right) d \cos \theta
$$

(2) The relative intensity is

$$
\frac{I\left(E_{0}, \mathbf{x}\right)}{I_{0}}=\int_{E_{c}}^{E_{0}} i\left(E_{0}, E, x\right) d E
$$

(3) The relative energy flux is

$$
\frac{E(x)}{E_{0}}=\frac{1}{E_{0}} \quad \int_{0}^{1} d \cos \theta \int_{E_{c}}^{E_{0}} E \cdot T\left(E_{0}, E, x, \cos \theta\right) d E
$$

(4) The integrated relative angular distribution is

$$
\mathbf{j}\left(\mathbf{E}_{0}, \mathbf{x}, \cos \theta\right)=\int_{\mathbf{E}_{\mathbf{c}}}^{\mathbf{E}_{0}} \mathrm{~T}\left(\mathrm{E}_{0}, \mathbf{E}, \mathbf{x}, \cos \theta\right) \mathrm{d} \cos \theta
$$

The isotropic incident case is given by integrating the initial distribution with respect to $\mathrm{d} \cos \theta_{0}$ with a weight function $\cos \theta_{0}$. 
Normalization of the above quantities corresponds to using 10,000 incident electrons in the calculations; i.e.,

$$
I_{0}=\int_{E_{i}}^{E_{0}} i\left(E_{0}, E, 0\right) d E=10^{4} \text {, and }
$$

$E_{c}$ is taken as $10^{-3}$. $E_{0}$ for all cases.

\section{Results}

Except for the angular dependence of back-scattering, the following results are based on the analysis of 2,000 Monte Carlo histories, with two cases of incident angular distribution (i.e., vertical and isotropic distribution), for initial energies of $20,10,5$, and $2.5 \mathrm{Kev}$. To show the effect of straggling, all calculations are done in two ways-one includes straggling and the other does not.

\subsection{Relative intensity, $\mathbf{I}(\mathbf{x}) / \mathbf{I}_{0}$}

The relative intensity is shown in Fig. $1(a-b)$ for $E_{0}=20$ and 2.5 Kev against depth $\mathbf{x}$. Full and dashed lines stand for the cases with straggling and without straggling, respectively. It should be noted that longer tails of intensity distribution due to straggling effects have been shown by several laboratory experiments (Grün, 1957, Frank, 1959) for vertical incidence.

\subsection{Energy flux $\mathrm{E}(\mathrm{x}) / \mathrm{E}_{\mathbf{0}}$}

Corresponding to Fig. $1(a-b)$, the energy flux is shown in Fig. $2(a-b)$ for $\mathbf{E}_{\mathbf{0}}=20$ and $2.5 \mathrm{Kev}$ against $\mathbf{x}$. Full and dashed lines stand for the cases with straggling and without straggling, respectively. 
- 3.3 Relative Energy Dissipation $\frac{1}{\mathbf{E}_{0}} \cdot \frac{\mathrm{dE}}{\mathrm{dx}}$

By differentiating the energy flux curve with respect to depth $x$, relative energy dissipation can be obtained as shown in Fig. 3 (a-b). From this we can see that the form of the energy dissipation curve changes with energy below $20 \mathrm{Kev}$. Therefore, the conventional as sumption of relative dissipation curves independent of energies is incorrect below $25 \mathrm{Kev}$.

\subsection{Angular dependence of back-scattering}

The ratio of back-scattered intensity (or energy flux) to incident intensity (or incident energy flux), is called the back-scattering coefficient or back diffusion coefficient (Frank, 1959; Wu, 1960). Experimentally, it is known that this coefficient is quite large for large $Z$ material (approximately $50 \%$ for $\mathrm{Pb}, Z=82$ ) but it is regarded as neglible for a low $Z$ material such as air. However, from satellite observations (O'Brien, 1962, for example), this does not seem as small as shown by laboratory experiments (Grün, 1957, Frank, 1959). Since laboratory data are limited to vertical (or normal) incidence only, it seems necessary to show the dependence of back-scattering on the incident angle, helpful in studying the so-called conjugation phenomena. Fig. 4 (a-b) shows angular dependence of the back-scattering coefficient for $\mathrm{E}_{0}=$ 20 and $2.5 \mathrm{Kev}$ with respect to intensity (particle flux) and energy flux. The horizontal lines in each figure correspond to isotropic incidence. 


\subsection{Energy dependence of back-scattering}

The results of Fig. 4 are replotted against energies in Fig. 5 (a, and b) for vertical incidence and isotropic incidence, respectively. It is well known that those coefficients decrease at high energies (Wu, 1960; Frank, 1959). Therefore, from the present results it can be concluded that the back-scattering of electrons has a maximum at an energy around $20 \mathrm{Kev}$, of the order of $7 \%$ for vertical incidence and more than $20 \%$ for isotropic incidence and that corresponding energy back-scattering is $4 \%$ and $14 \%$ for vertical and isotropic incidence, respectively.

It is interesting to see that the effect of straggling is reversed around $10 \mathrm{Kev}$ for the isotropic incidence. At any rate, a decrease of back-scattering at low energies can be explained as follows: because of the spread in energy distribution after several collisions the amount of low energy electrons increases and these low energy electrons are unable to leave the air even though they are back scattered. On the other hand, a decrease of back-scattering at a high energy is simply due to the fact that the transformed distribution in the laboratory system is concentrated forward, even though scattering distributions in the center of mass system of colliding particles are regarded as isotropic.

\subsection{Application for auroral electrons}

Fig. $6(a, b)$ shows the intensity and energy flux distribution of monoenergetic $20 \mathrm{Kev}$ electrons in the atmosphere (CIRA, 1961) for vertical and isotropic distribution, respectively.

Fig. $7(\mathrm{a}, \mathrm{b})$ shows similar curves for $2.5 \mathrm{Kev}$ electrons. 
It is seen that the effect of straggling is negligible and even reversed below $5 \mathrm{Kev}$, whereas above $20 \mathrm{Kev}$ the distribution of penetrating electrons due to straggling is significantly increased and nearly $1 \%$ exceed the range given by a continuous energy loss formula. Differentiating these curves with respect to altitude $\bar{Z}$, gives the vertical distribution of energy dissipation of auroral electrons. Fig. 8 shows one example for $20 \mathrm{Kev}$ electrons. Here again the effect of straggling is significant. It should be noted that not only the most penetrating depth but also the height of maximum dissipation shifts to a lower altitude, i.e., to a deeper atmosphere.

Changes of angular distributions with altitude are shown in Fig. $9(\mathrm{a}, \mathrm{b})$ for mono-energetic $20 \mathrm{Kev}$ electrons where relative intensities normalized to the initial intensity are plotted against the cosines of angles from nor mal directions. The change of angular distribution is similar for different energies as far as they are expressed with respect to $x$. It should be noted that angular distributions rapidly approach perfect diffusion with cosine square distribution, rather than Gaussian distribution, around half of the penetrating depth (Wu, 1960) for both cases of incidence distribution (vertical and isotropic). For a comparison cosine square curve is drawn in both cases. Fig. $10(a, b)$ shows the plotting of a figure similar to Fig. 9 with the angle from the normal direction as a parameter. It should be noted that there is a maximum height (i.e., depth) in the intensity for oblique direction in the case of normal incidence, while intensity in the vertical direction decreases monotonically in both cases. 
Finally, Fig. 11 shows the change of the energy spectrum of monoenergetic $20 \mathrm{Kev}$ electrons with altitude. Full and dashed lines correspond to vertical and isotropic incidence, respectively. In the bottom, energies correspond to continuous energy loss, i.e.

$$
E_{\mathrm{m}}=\mathrm{E}_{0}-\cdot-\int_{0}^{\mathrm{x}} \frac{\mathrm{dE}}{\mathrm{dx}}, \mathrm{dx^{ \prime }}
$$

It is interesting to note that the energies corresponding to the maximum intensities at each altitude (or depth) are considerably higher than $\mathbf{E}_{\mathbf{m}}$.

\section{Conclusion}

From the results of the present calculations we can draw the following conclusions.

(1) As seen from Fig. 1, 2, and 11, the values for the intensities and energy fluxes of electrons, based on conventionally used rangeenergy relations, are significantly underestimated.

(2) Because of the straggling effect, not only the depth of the tail of the penetrating electrons (Figs. 6 and 7), but also the depth of maximum intensity, shifts toward a deeper atmosphere, i.e., a lower altitude for a given energy of incident electrons.

(3) The back-scattering coefficient decreases rapidly below $10 \mathrm{Kev}$ (Fig. 5). Since this coefficient also decreases at high energies, we can 
- conclude that back-scattering is largest around $20 \mathrm{Kev}$ (between 10 and $25 \mathrm{Kev*)}$ which is the order of $7 \%$, and $20 \%$ for vertical and isotropic incidence, respectively for intensity, nearly half of these values for energy flux.

\section{ACKNOW LEDGMENTS}

The FORTRAN programs are prepared by M. J. Berger and S. M. Seltzer in the National Bureau of Standards under contract R-80 between NASA and the National Bureau of Standards. A more detailed report including the discussion of energies above $25 \mathrm{Kev}$ will be published later as a NASA Technical Note.

Table I

\begin{tabular}{|c|c|c|}
\hline$E_{0} \mathrm{Kev}$ & $\mathrm{r}_{0} \mathrm{~g} \mathrm{~cm}^{-2}$ & $Z_{0} \mathrm{Km}$ \\
\hline 20 & $9.824 \cdot 10^{-4}$ & 93.24 \\
10 & $2.909 \cdot 10^{-4}$ & 100.18 \\
5 & $8.79 \cdot 10^{-5}$ & 107.13 \\
2.5 & $2.76 \cdot 10^{-5}$ & 118.02 \\
\hline
\end{tabular}

*The value at $25 \mathrm{Kev}$ is slightly less than that at $20 \mathrm{Kev}$. 


\section{REFERENCES}

Berger, M. J.

Berger, M. J. and

Seltzer, S. M.

Berger, M. J. and

Seltzer, S. M.

Blunck, O. and

Leisegang, S.

Frank, H.

Goudsmit, S. and

Saunderson, J. L.

Grün, A. E.

Kallman-Bijl, H. ,

Boyd, R. L. F., Lagow,

H., Poloskov, S. M. and

Priester, W.

Landau, L.

Maeda, K.

Maeda, K.

Maeda, K.

Maeda, K. and

Singer, S. F.

Moliere, G.

Mott, N. F.

O'Brien, B. J.

Perkins, J. F.
1963 Methods in Computational Physics, V. I, pp. 135-215, Academic Press, New York

1964a NASA Special Rept. SP-3008

1964b Nat. Acad. Sci.-Nat. Res. Council, Publ. No. 1133 (Rept. No. 101)

1950 Zeitschrift für Phys, 128, 500

1959 Z. Natfasch 14A, 247

1940 Phys. Rev. 57, 24

1957 Z. Naturforsch $12 \mathrm{~A}, 89$

1961 Cospar International Reference Atmosphere (C.I.R.A), Interscience, New York

1944 J. Phys. (Acad. Sci. U.S.S.R.) 8,201

1962 NASA Tech. Rept. TR-R-141

$1963 a$ J. Geophys. Res. 68, 185

1963b Proc. International Sym. Strato. Mesopheric Circulation 36, 451

1961 Arkiv Fysik 조 531

1948 Zeits. f. Naturfors. 3A, 78

1929 Proc. Roy. Soc. Al24, 425

1962 Z. Natfasch 14A, 247

1962 Phys. Rev. 126, 1781 
Rees, M. H. $1963 \quad$ Planet. Space Sci. 11, 1209

Rohrlich, F., and 1954 Phys. Rev. 93, 38

Carlson, B. C.

Rossi, B.

1952 High Energy Particles, p. 440,

Prentice-Hall, New York

Spencer, L. V.

1959 Nat. Bur. Standards Monograph 1

$\mathrm{Wu}, \mathrm{C} . \mathrm{S}$.

1960 Nuclear Spectroscopy Part A, pp. 15-30, Academic Press,

New York 


\section{CAPTIONS}

Figure 1-Relative intensities of penetrating the mono-energetic electrons in $\operatorname{air} I(x) / I_{0}$ vs. depth of air $x$, where $x=r / r_{0}$ with $r$ in $g / \mathrm{cm}^{2}$ and $r_{0}$ is the practical range of electrons in $\mathrm{g} / \mathrm{cm}^{2}$, corresponding to the initial energy $E_{0}$ as shown in Table I

$$
\text { (a) } E_{0}=20 \mathrm{Kev}, \text { (b) } E_{0}=2.5 \mathrm{Kev}
$$

The full and dashed lines stand for the calculations with straggling and without straggling, respectively. Thick lines and thin lines correspond to isotropic incidence and vertical incidence respectively.

Figure 2-Relative energy fluxes of mono-energetic electrons $E(x) / E_{0}$ vs. the depth of air $x$, corresponding to the different initial energies shown in Figure 1. All notations are the same as in Figure 1.

Figure 3-Relative energy dissipation curves of mono-energetic electrons $\left(\triangle \mathrm{E} / \mathrm{E}_{0}\right) /\left(\Delta \mathrm{r} / \mathrm{r}_{0}\right)$ vs. depth of air $\mathrm{x}$, corresponding to different initial energies shown in Figures 1 and 2. All notations are the same as those used in Figures 1 and 2.

Figure 4-Back-scattering vs. incident (or incident pitch) angle of a mono-energetic electron beam. Full and dashed lines stand for the calculations with straggling and without straggling, respectively. Thick lines and thin lines correspond to particle flux (intensity) and energy flux, respectively.

Figure 5-Back-scattering coefficients vs. of incident energy. The two figures ( $a$ and $b$ ) correspond to two cases for incident angles of monoenergetic electrons, i.e., (a) vertical incidence, (b) isotropic incidence. 
* Figure 6-Relative intensity (particle flux) and relative energy flux of electrons with an initial energy of $20 \mathrm{Kev} v \mathrm{~s}$. altitude (in $\mathrm{km}$ ) in the CIRA (1961) model atmosphere. The height indicated by $Z_{0}$ corresponds to the maximum penetration depth corresponding to practical range for the initial energy of electron $E_{0}=20 \mathrm{Kev}$. Full and dashed lines correspond to calculations with straggling and without straggling, while thick lines and thin lines stand for intensity and energy flux, respectively, (a) vertical incidence, (b) isotropic incidence.

Figure 7-Intensity and energy flux distribution of mono-energetic 2.5 Kev electrons. All notations are the same as in Figure 6.

Figure 8-Energy dissipation curves for electrons with initial energy $\mathbf{E}_{0}=20 \mathrm{Kev}$ in the atmosphere, CIRA (1961) model.

Figure $9(\mathrm{a}, \mathrm{b})$-Angular distribution (relative intensity/steradian) electrons with initial energy $E_{0}=20 \mathrm{Kev}$. The symbol $\mathrm{x}$ indicates nondimensional atmospheric depth $\mathrm{r} / \mathrm{r}_{0}$ where $\mathrm{r}_{0}=9.824 \times 10^{-4} \mathrm{~g} / \mathrm{cm}^{2}$. (A) vertical incidence, (b) isotropic incidence.

Figure $10(\mathrm{a}, \mathrm{b})$-Plots similar to Figure 9 for relative intensity against nondimensional depth $\mathbf{x}$ for different zenith (or pitch) angles $\theta$. (a) ver tical incidence, (b) isotropic incidence.

Figure 11-Differential energy spectrum of initially mono-energetic electrons, $\mathbf{E}_{0}=20 \mathrm{Kev}$, in the atmosphere. Altitudes shown in parenthesis are the altitudes in CIRA (1961) atmosphere, corresponding to nondimensional depth $\mathbf{x}$.

$E_{\mathrm{m}}$ shown in the bottom corresponds to the energy given by the conventional energy-range relation i.e.,

$$
E_{m}=E_{0}-\int_{0}^{x} \frac{d E}{d x^{\prime}} d x^{\prime}
$$


where $d E / d x^{\prime}$ is given by the Bethe formula as a function of energy.

Full lines and dashed lines stand for the vertical and isotropic distributions of incident electrons, respectively. 

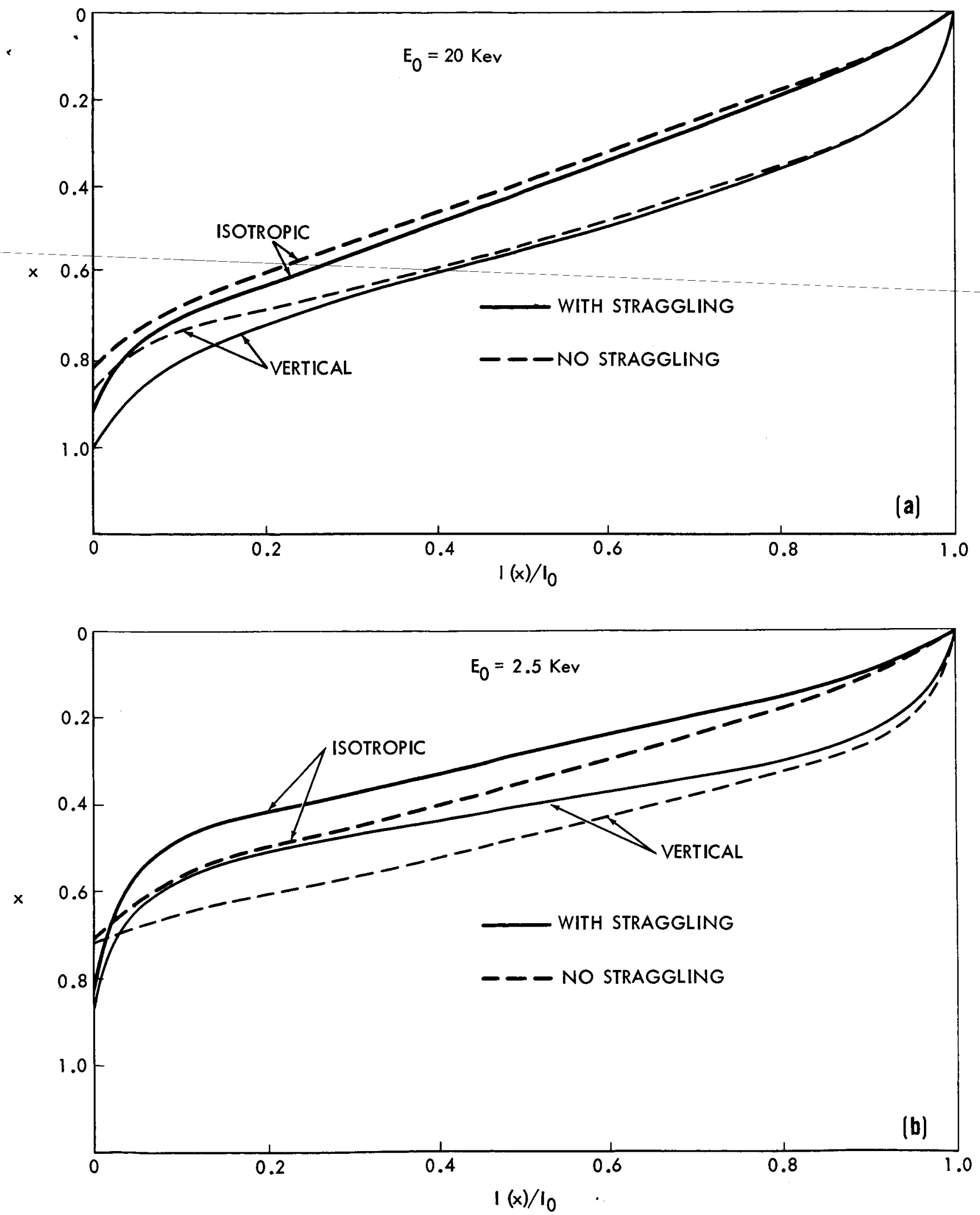

FIGURE 1 

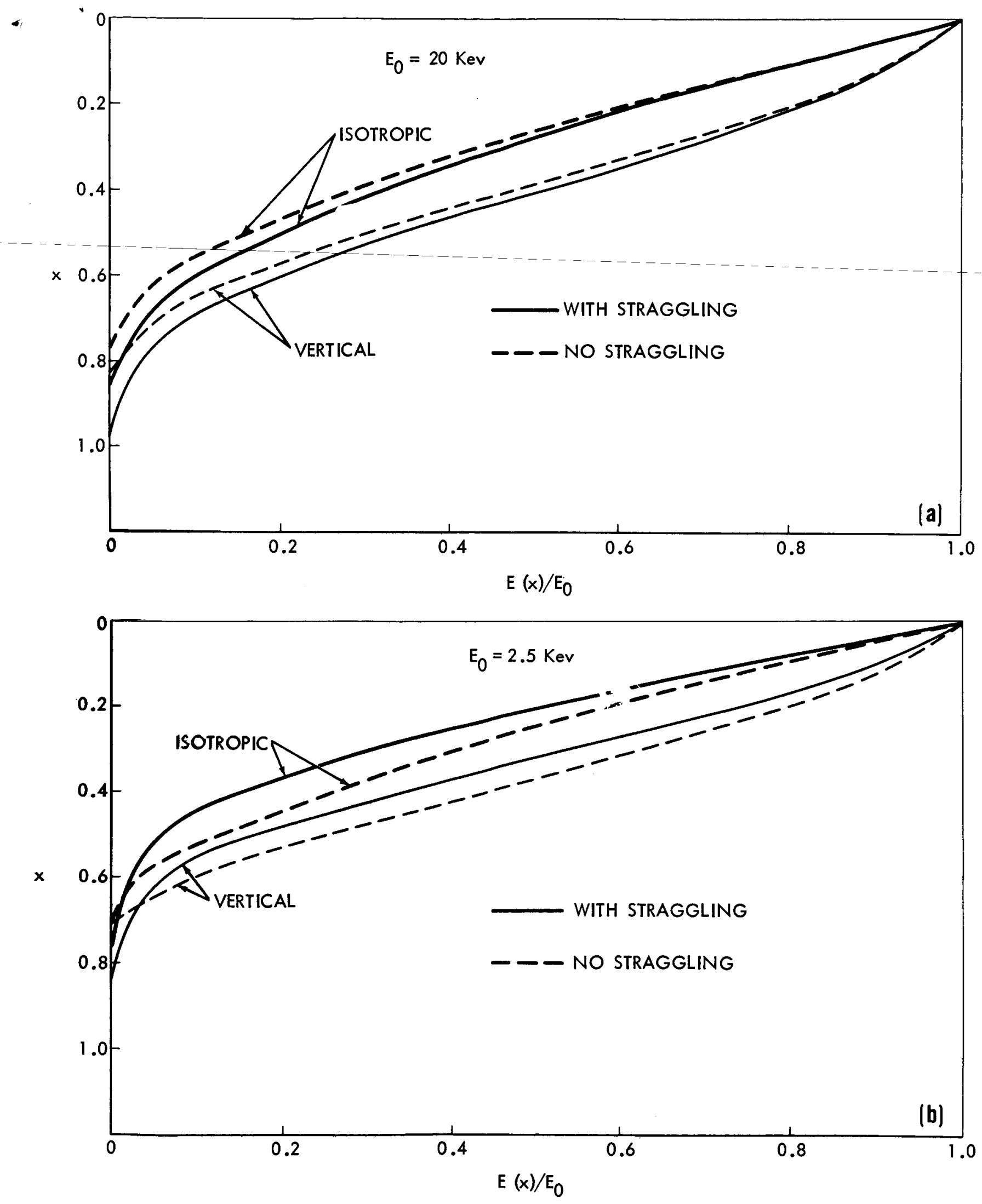

FIGURE 2 

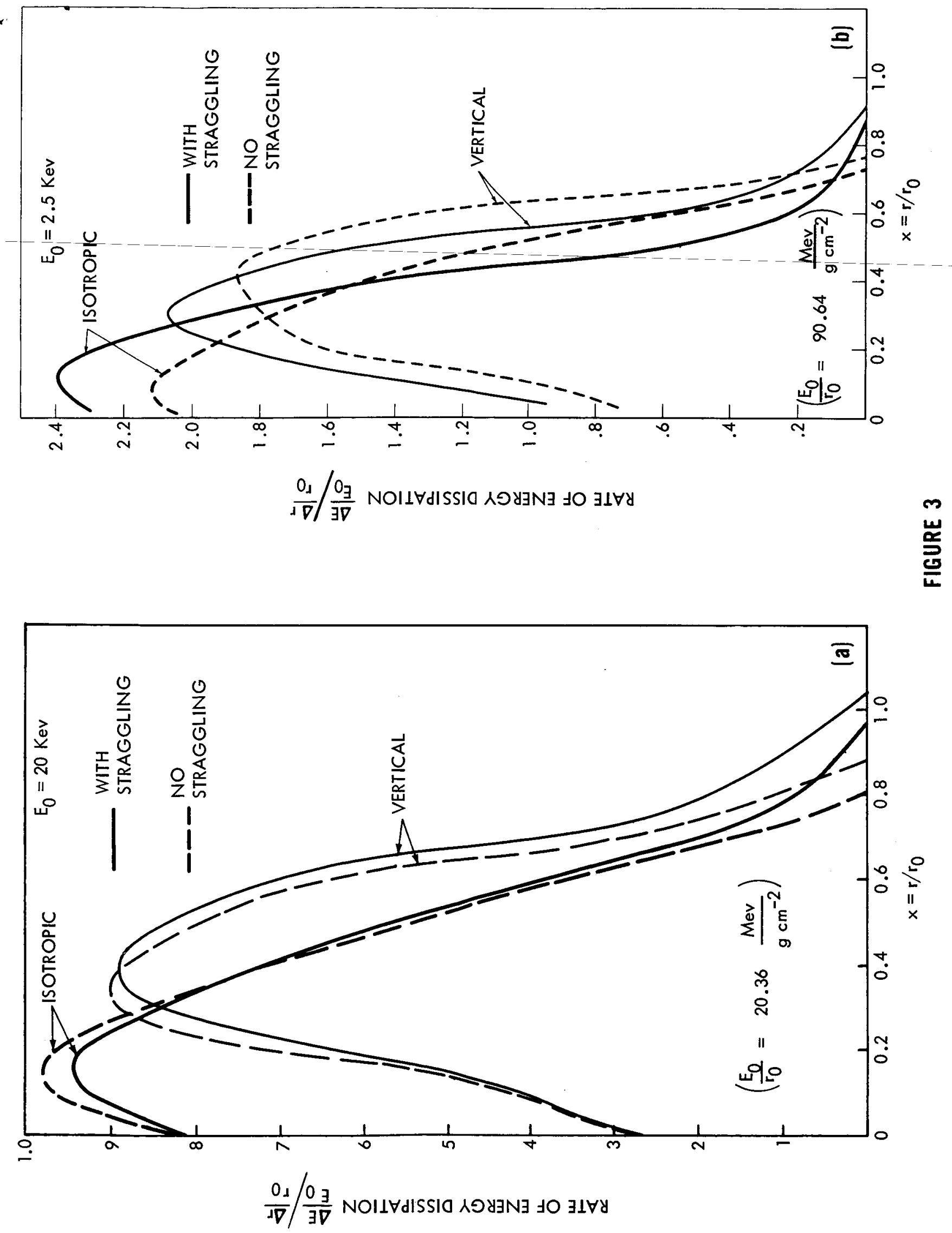


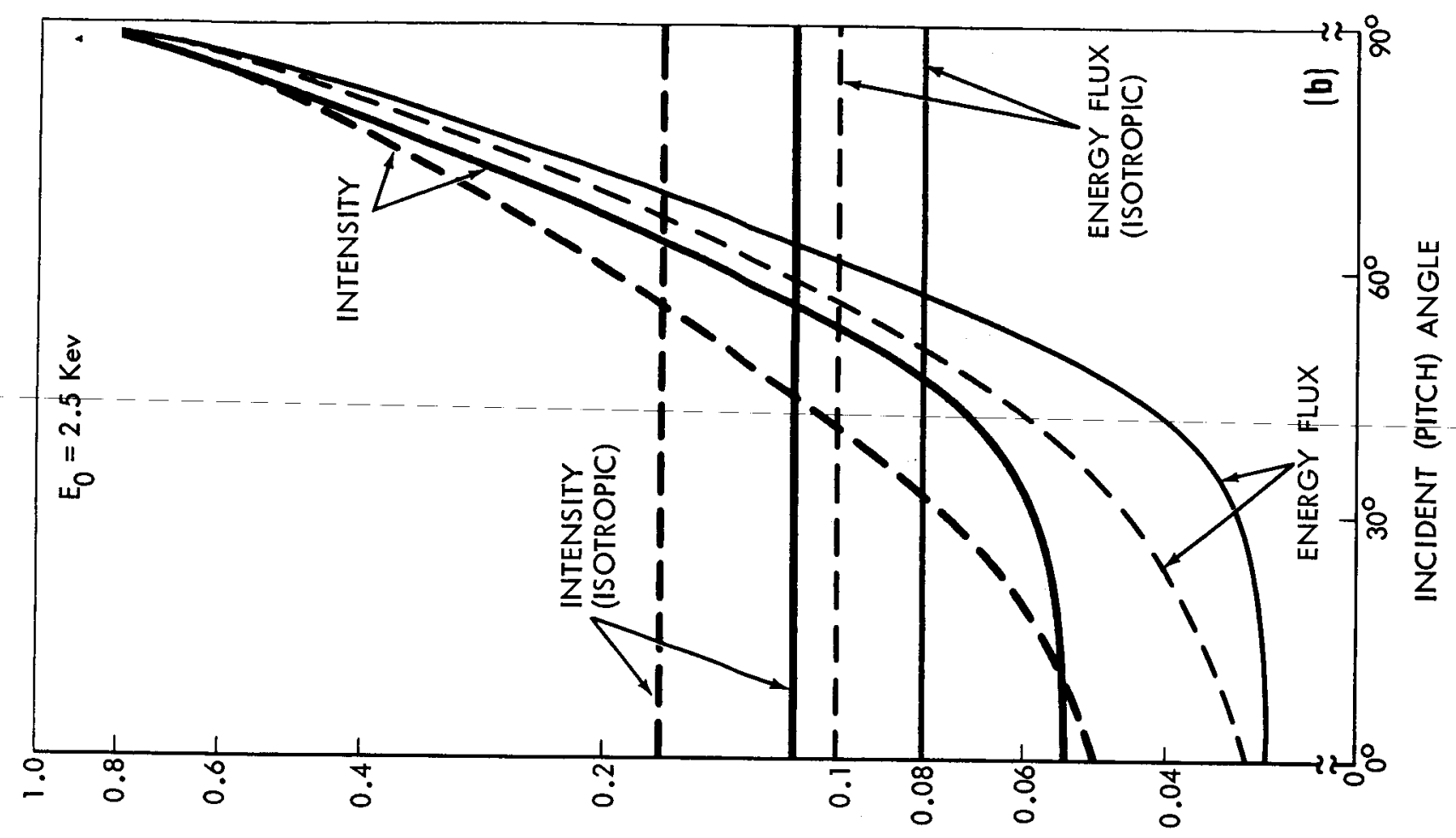

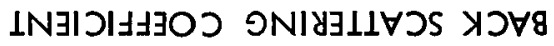

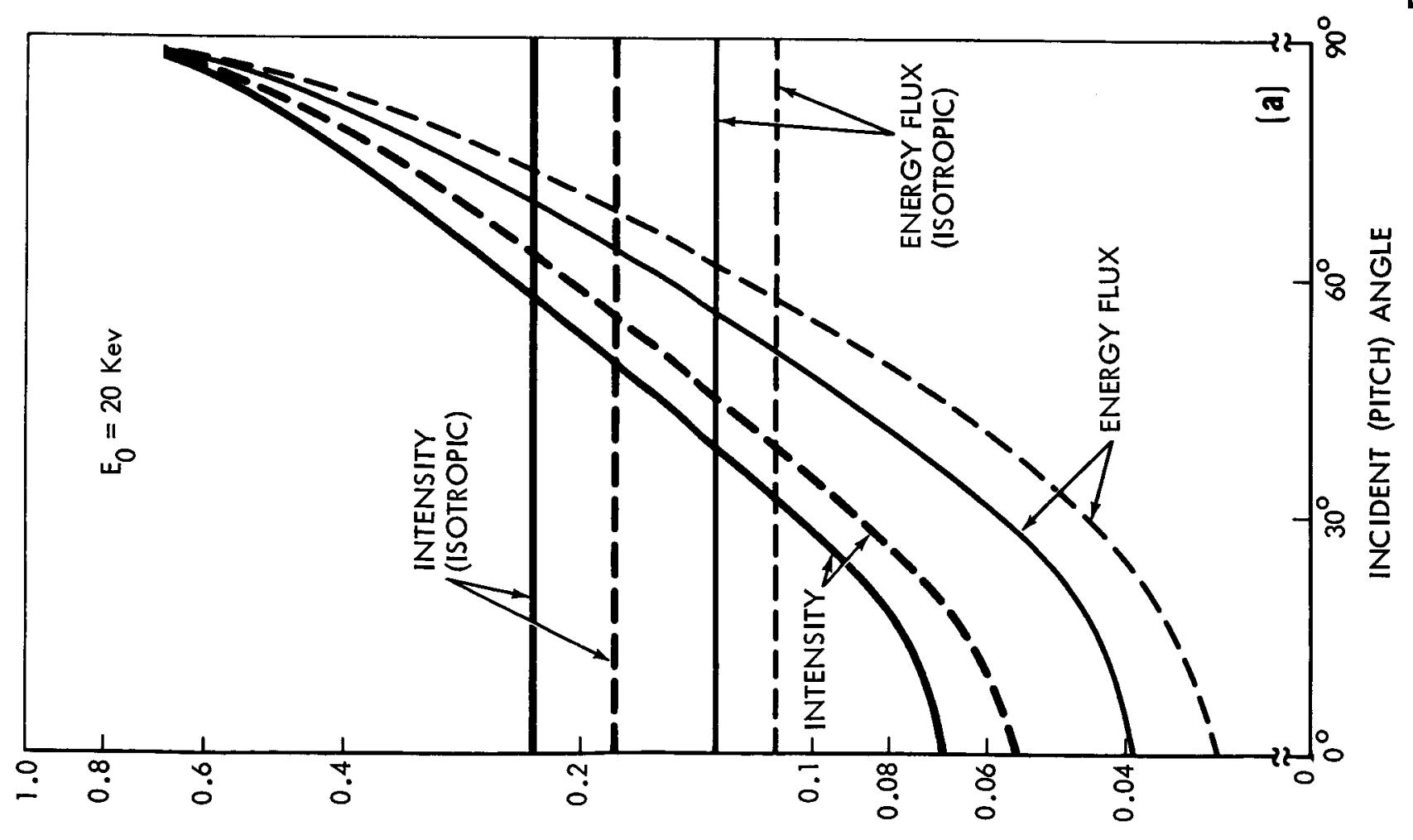

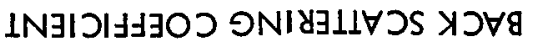




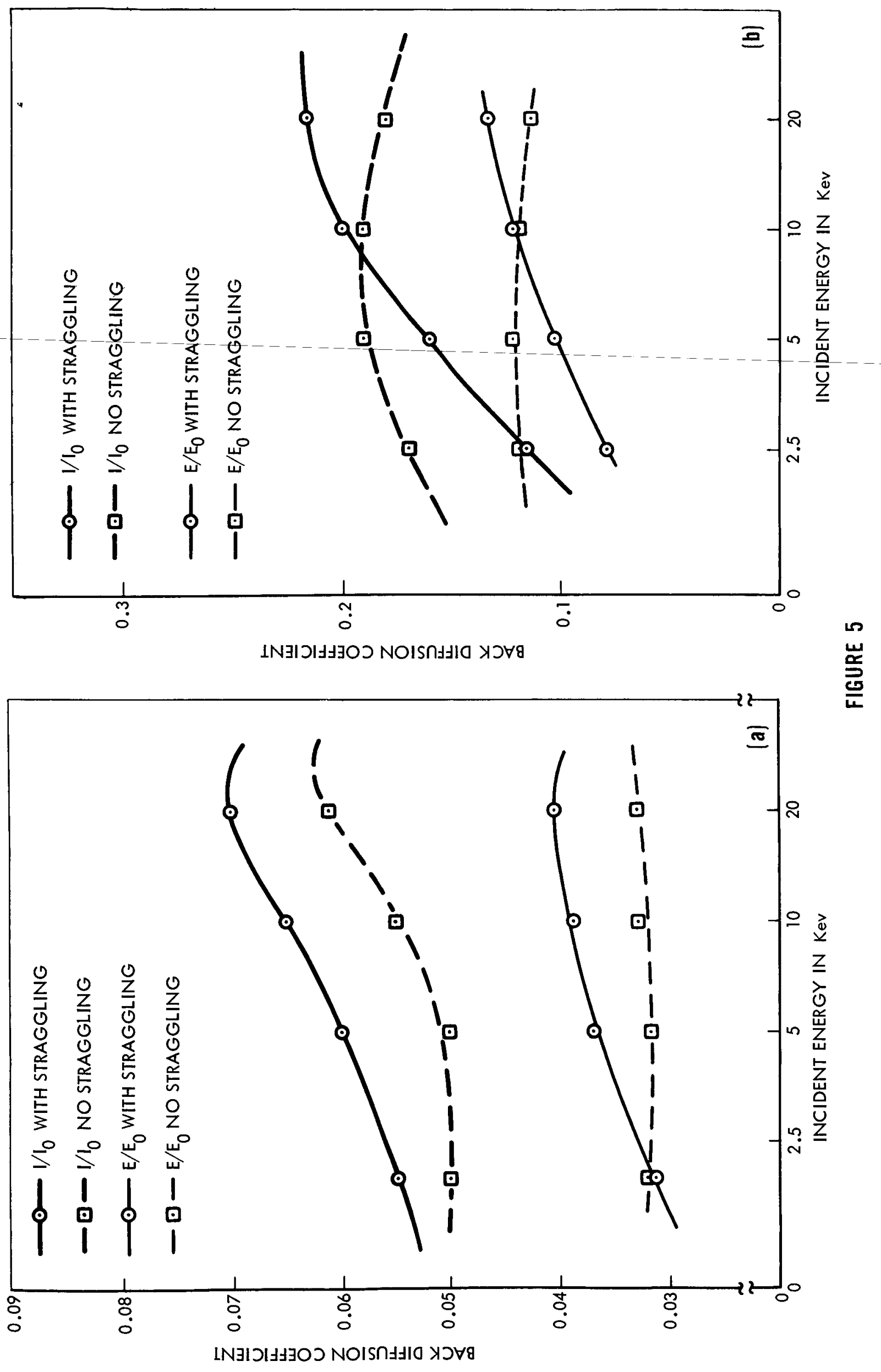



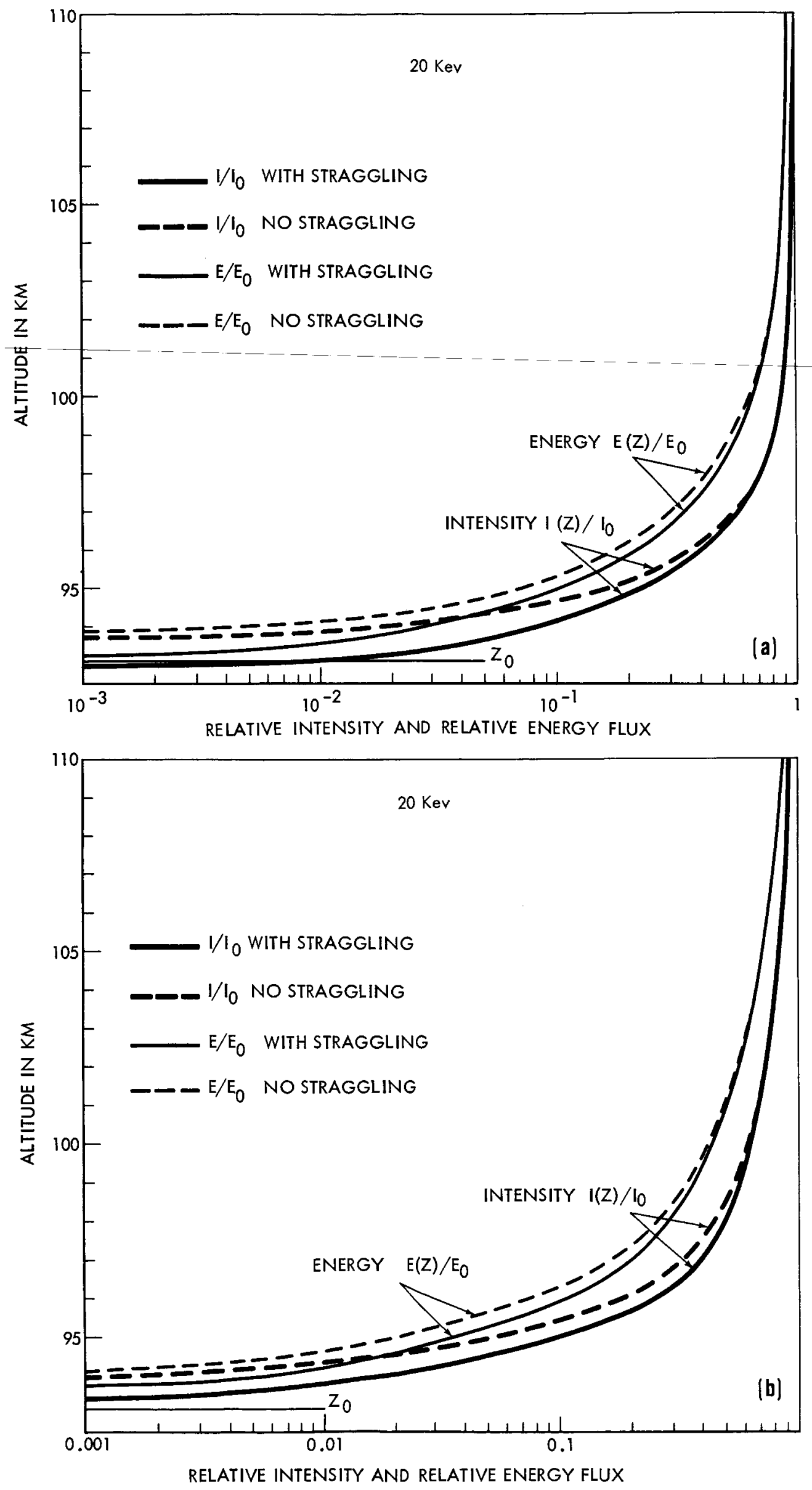

FIGURE 6 

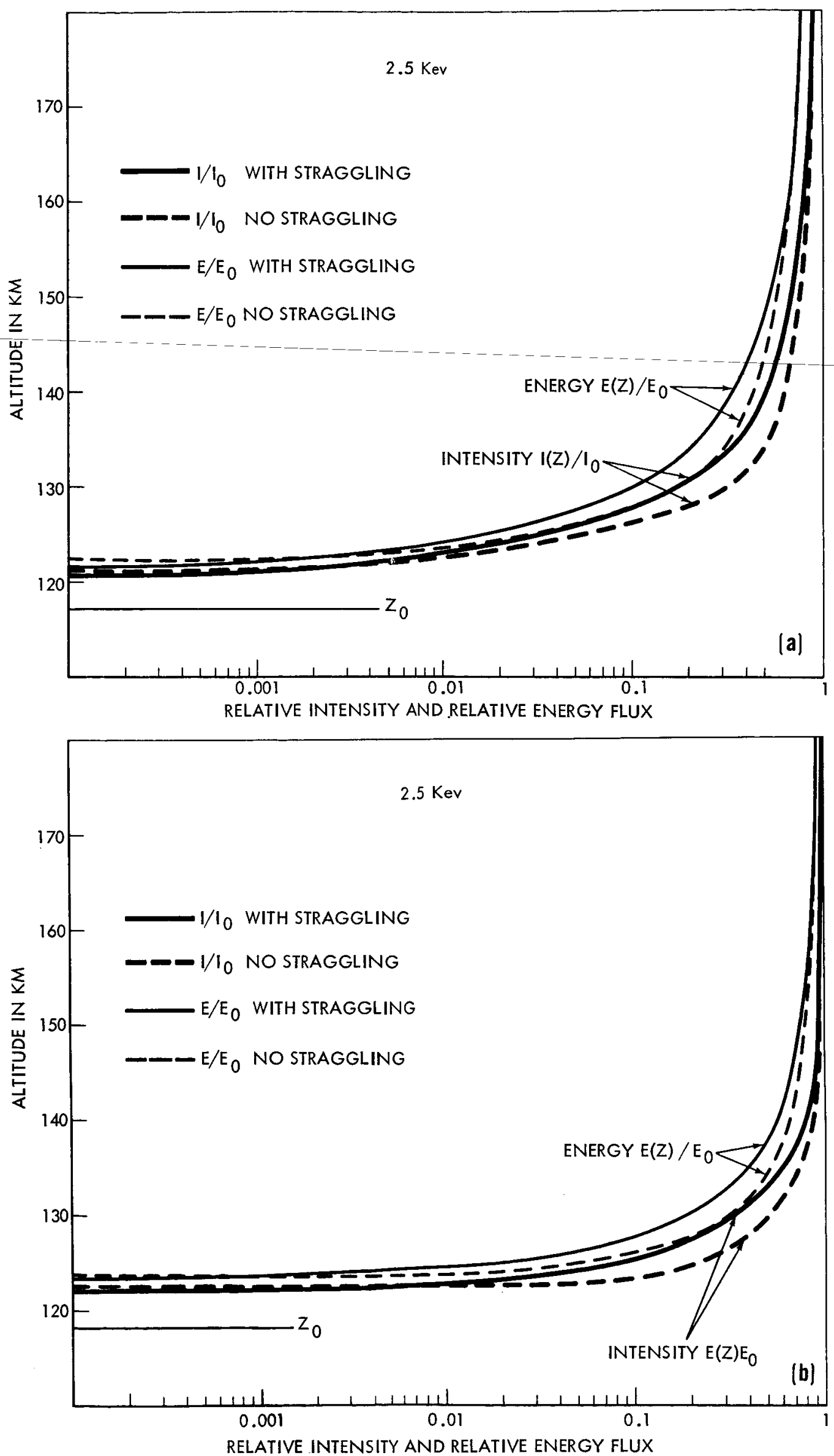

FIGURE 7 


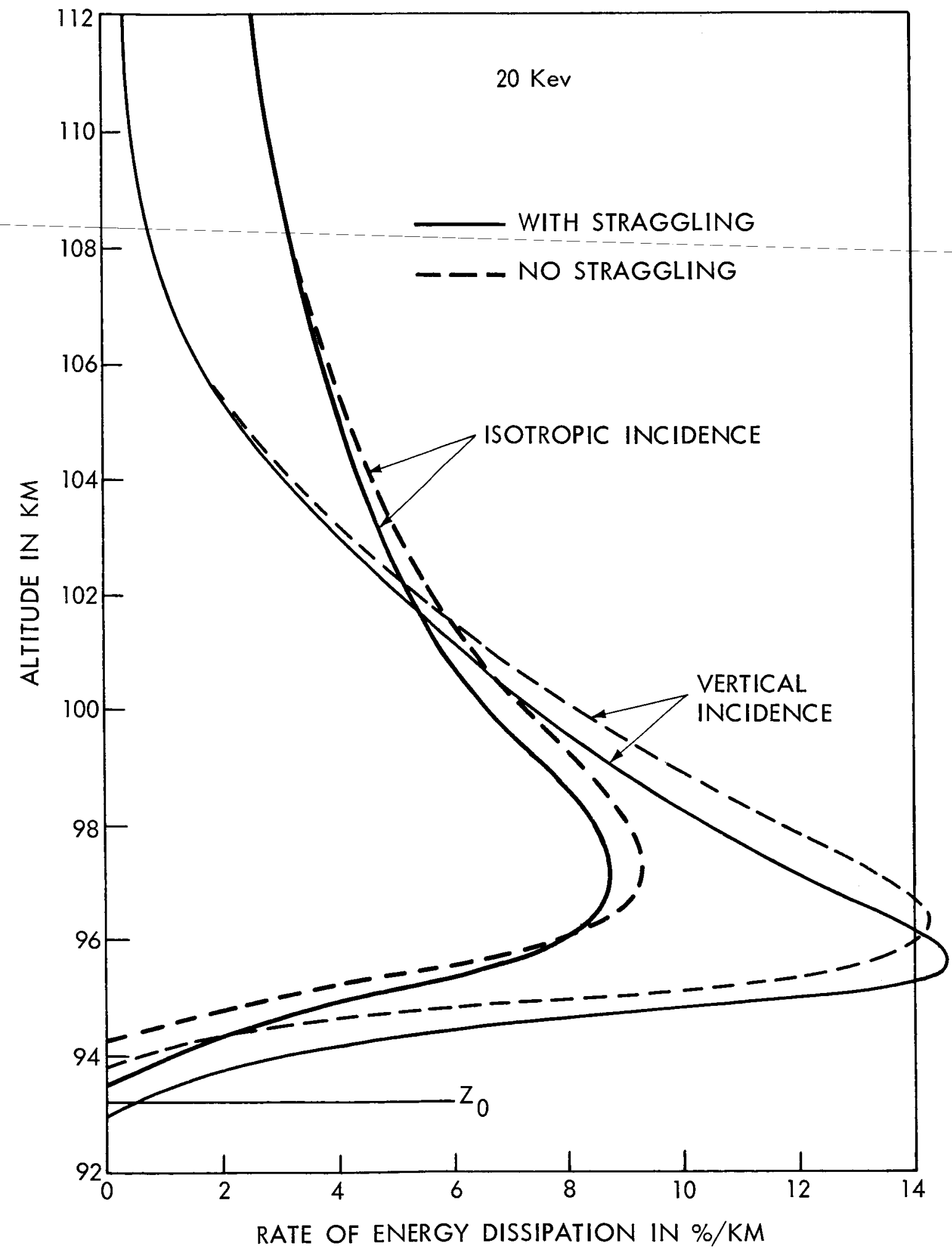

FIGURE 8 


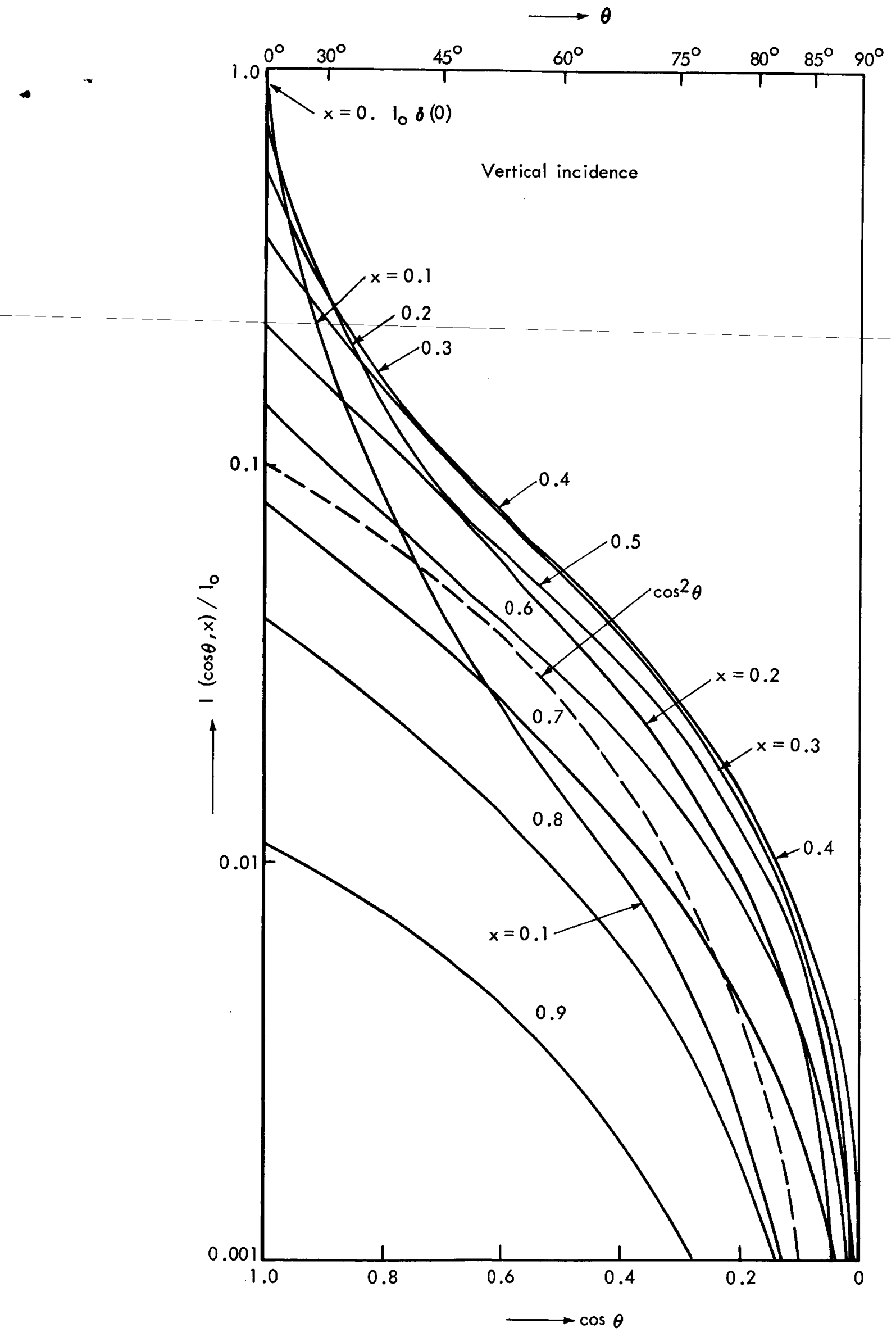

FIGURE 9 (a) 


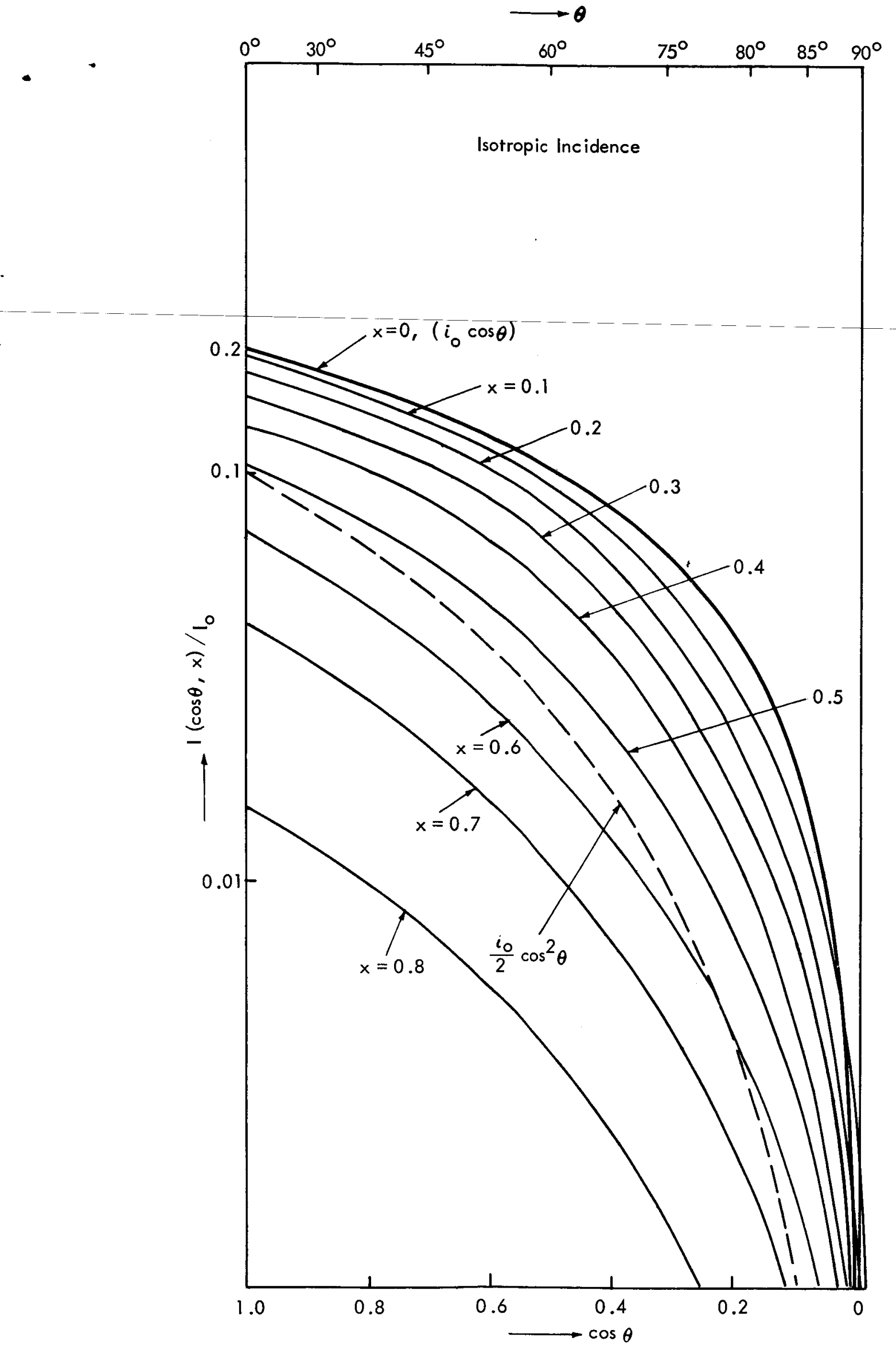

FIGURE 9 (b) 


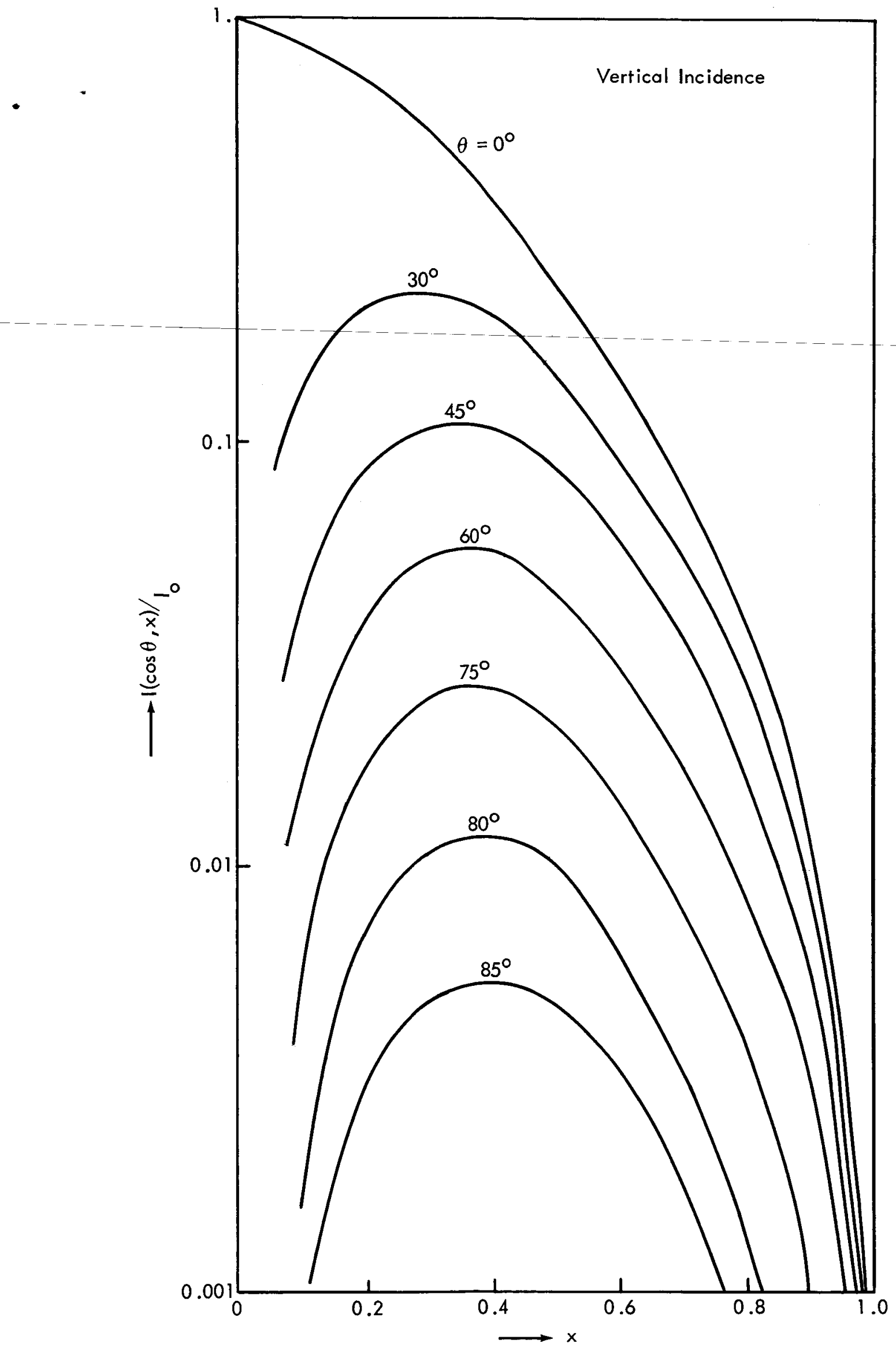

FIGURE 10 (a) 


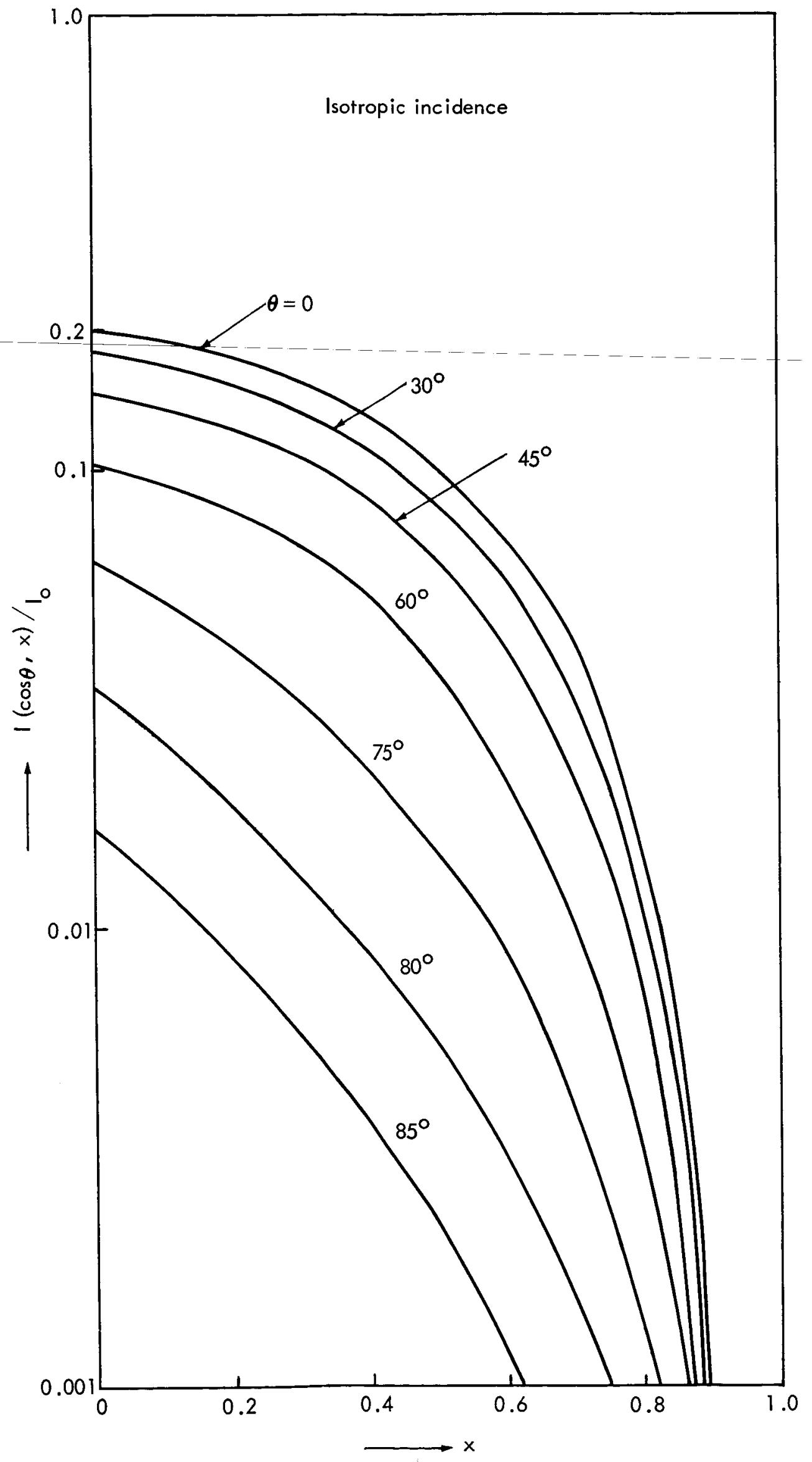

FIGURE 10 (b) 


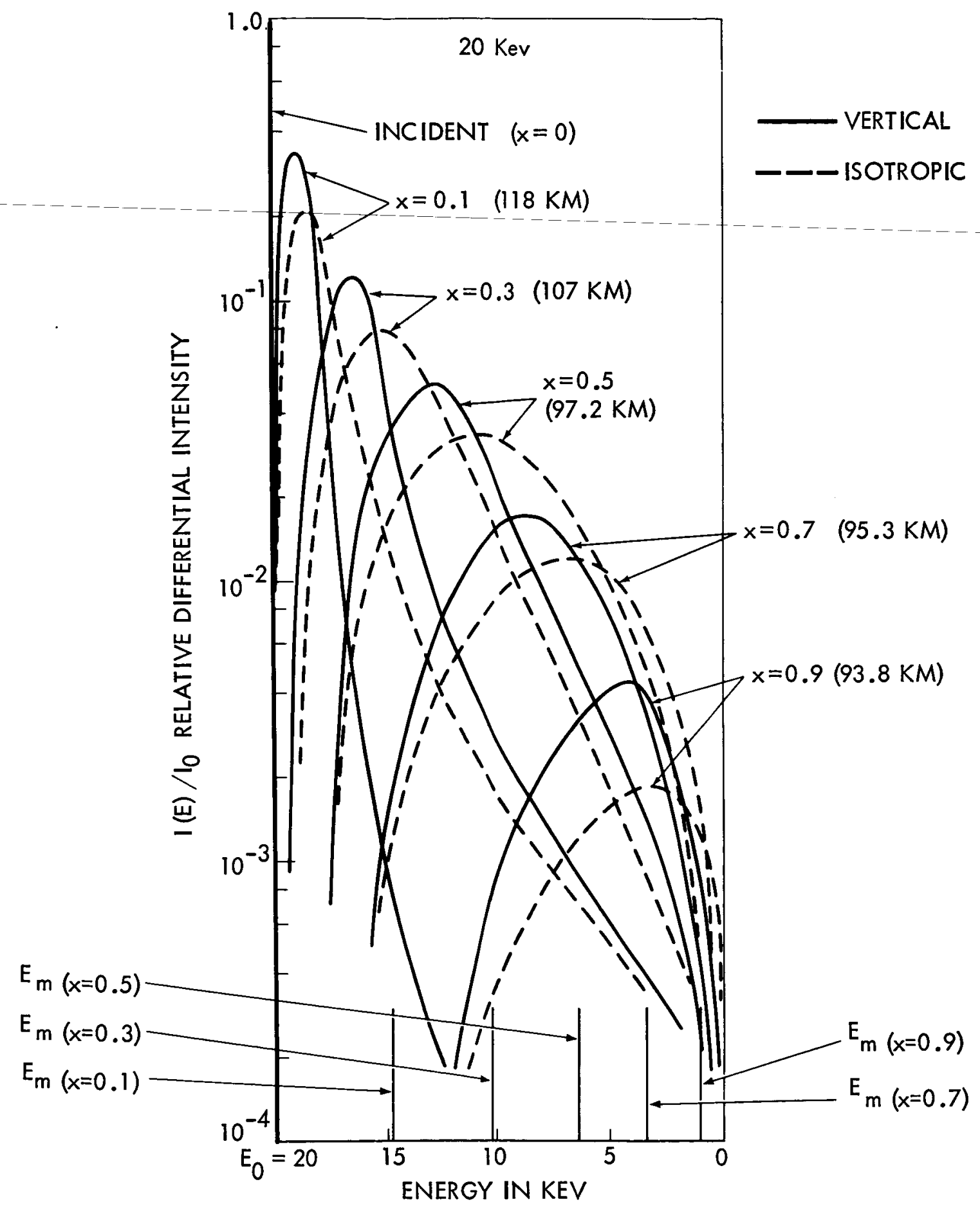

FIGURE 11 